\title{
Meet the Challenges of Mass Vaccination against COVID-19
}

\author{
Zheng Chen ${ }^{1}$ and Lanjing Zhang ${ }^{2,3 *} \odot$ \\ ${ }^{1}$ Bristol Myers Squibb, Co., Summit, NJ, USA; ${ }^{2}$ Department of Pathology, Princeton Medical Center, Plainsboro, NJ, USA; \\ ${ }^{3}$ Department of Biological Sciences, Rutgers University, Newark, NJ, USA
}

\begin{abstract}
As coronavirus disease 2019 (COVID-19) vaccines continue to be reviewed and approved by regulatory bodies for emergency use, mass vaccination against COVID-19 is on the horizon. This will significantly reduce the incidence and mortality of COVID-19. However, the unintended consequences of mass vaccination and their associated challenges could be urgent and severe. To effectively prepare for and meet these challenges, we propose three guiding principles and share cautionary perspectives on the mass vaccination against COVID-19. These opinions could help shape policy-making and the implementation of the mass vaccination.
\end{abstract}

Three coronavirus disease 2019 (COVID-19) vaccines have been reported to show $>90 \%$ efficacy. ${ }^{1-3}$ Two vaccines against COVID-19 have been approved for emergency use in the USA, and one vaccine has been approved in the UK. ${ }^{4-8}$ The frameworks for the optimal allocation of COVID-19 vaccines have been well planned, and should satisfactorily address allocation issues.,10 However, the unintended consequences of mass vaccination and their associated challenges might be urgent and severe. They should be carefully prepared for and prevented. Here, we propose three guiding principles and share a cautionary perspective on mass vaccination.

Three principles are recommended for the effective conduct of mass vaccination against COVID-19, which include accountability, transparency, and immediacy. Accountability is required for effective governance, quality control, and root-cause analysis, which could identify the causes of any adverse events associated with the vaccination; transparency is required to reduce misinformation, build trust among stakeholders and increase vaccination rates; immediacy is required because of the rapidly changing vaccine development landscape (e.g., 9 days between the interim and final reports for a vaccine's efficacy), ${ }^{11}$ and the rapidly increasing number of COVID-19 cases.

A proposed action plan to meet the challenges of mass vaccination

First, actively monitor the efficacy and safety of the new vaccines

Keywords: Adverse events; Clinical trial; COVID-19; Vaccine.

Abbreviations: COVID-19, coronavirus disease 2019; IgG, Immunoglobulin G; SARS-CoV-2, severe acute respiratory syndrome coronavirus 2 .

Received: December 14, 2020; Revised: December 24, 2020; Accepted: December 25,2020

*Correspondence to: Lanjing Zhang, Department of Pathology, Princeton Medical Center, Plainsboro, NJ, USA. ORCID: https://orcid.org/0000-0001-5436-887X. Tel: +1-609-853-6833, Fax: +1-609-853-6841, E-mail: lanjing.zhang@rutgers.edu

How to cite this article: Chen Z, Zhang L. Meet the Challenges of Mass Vaccination against COVID-19. Exploratory Research and Hypothesis in Medicine 2020; 000(000):000-000. doi: 10.14218/ERHM.2020.00075. at local sites. As noted previously, ${ }^{12,13}$ vaccine safety cannot be thoroughly assessed easily. In addition, stringent storage requirements mean that the vaccines are susceptible to degradation. ${ }^{13}$ Given the unprecedented number of vaccinations required in a short time, local healthcare providers or authorities should keep track of or regular contact with vaccinated individuals about the vaccine's efficacy and safety. In addition, it is recommended that regular and appropriate communications among vaccine administration sites, regulators, and local health authorities. This will help to resolve any issue associated with a vaccine batch and lot numbers, which could be timely notified and systematically corrected.

Second, timely publish the full and in-depth data from the vaccine trials. As the regulatory bodies review the vaccine manufacturers' application for approval, the public and healthcare providers require this trial data, and regular updates on the additional trial results and postmarketing data (i.e., phase 4 trial), to make more informed decisions for the vaccination and laboratory tests.

Third, maintain the implementation of nonpharmaceutical interventions. Vaccine production might take 12-24 months to reach full capacity. ${ }^{14}$ An additional 28 days might be required to complete the vaccination protocol for some vaccines. ${ }^{1,2}$ Moreover, approximately $25 \%$ of the public would refuse the vaccination when it is available, ${ }^{15-17}$ which is close to or lower than the vaccination rate (75-90\%) required for herd immunity ${ }^{14}$ and might cause delayed or unsuccessful herd immunity. Therefore, the development of herd immunity will probably require $13-25$ months if successful at all, during which nonpharmaceutical interventions are required to control COVID-19. In addition, $5-10 \%$ of the vaccinated will not be protected as the trial data show. $1,2,11$ Before understanding who will not be protected by the vaccine(s), nonpharmaceutical interventions probably should be implemented to protect this $5-10 \%$ of the vaccinated.

Fourth, prepare for a drug shortage owing to the mass production of COVID-19 vaccines. We anticipate that many manufacturing facilities will be rightfully allocated to mass vaccine production. Subsequently, these facilities cannot be used for their originally planned drug production, which could lead to a shortage of certain drugs. Pharmacies and healthcare providers should prepare for the possible shortage and seek alternative drugs for the continued care 
of the patients. However, no data or formal communication have been released for this. Therefore, we call for drug manufacturers to timely release the related information, and to optimize the allocation process; therefore, alternative drugs will be available.

Fifth, interpret the severe acute respiratory syndrome coronavirus 2 (SARS-CoV-2) serology results with caution. The ChAdOx1 nCoV-19 vaccine is effective in generating anti-spike SARSCoV-2 Immunoglobulin G (IgG) in $90 \%$ of vaccinated individuals, ${ }^{1}$ which could lead to positive results in the SARS-CoV-2 IgG test. However, unless the postvaccination IgG level is assessed in every vaccinated individual (not considering the test's false positivity), we cannot reliably distinguish a positive SARS-CoV-2 IgG result owing to vaccination versus the infection in a vaccinated individual that does not generate IgG and would not be protected. In addition, no data is currently available regarding the protective level of IgG. Therefore, caution must be used to interpret SAR$\mathrm{CoV}-2$ serology results regarding infection status, and the molecular testing of the SARS-CoV-2 virus in nasopharyngeal specimens should be used to examine infection status.

Sixth, be aware of the ineffectiveness of the vaccines. The vaccinated individuals might not have the required SARS-CoV-2 antibodies for several reasons: during the antibody generation window period (28 days for ChAdOx $1 \mathrm{nCoV}-19),{ }^{1}$ lack of efficacy (5-10\% of the trial population $),{ }^{1-3}$ possible decline in antibodies (12-18 months postvaccination), ${ }^{14}$ and virus mutations. ${ }^{18}$ Therefore, the vaccinated individuals will probably be protected against COVID-19, but could still be infected. Further, the immunogenicity data from the other two vaccine trials remain largely unknown. ${ }^{2,3}$ Therefore, it is difficult to predict the possibility of infection in the individuals who have received these vaccines. Therefore, vaccinated individuals should remain cautious and maintain the use of nonpharmaceutical interventions.

Finally, maintain sufficient resources for COVID-19 patients, such as healthcare providers, medical equipment, and medical supplies. The mass vaccination could help control the COVID-19 pandemic, but there are already approximately 75 million COVID-19 cases in the world. ${ }^{19}$ Therefore, it is probably warranted that the required resources for COVID-19 patients should be provided to reduce the COVID-19 mortality rate, in particular, when a large number of resources are allocated for mass vaccination.

\section{Acknowledgments}

The opinions expressed here are solely those of the authors, and not of their affiliations.

\section{Funding}

None.

\section{Conflict of interest}

ZC is an employee and owns stocks of Bristol Myers Squibb, Co. The spouse of LZ is an employee and owns stocks of Bristol Myers Squibb, Co.

\section{Author contributions}

The two authors conceptualized, wrote, and edited the manuscript.

\section{References}

[1] Ramasamy MN, Minassian AM, Ewer KJ, Flaxman AL, Folegatti PM, Owens DR, et al. Safety and immunogenicity of ChAdOx1 nCoV-19 vaccine administered in a prime-boost regimen in young and old adults (COVO02): a single-blind, randomised, controlled, phase 2/3 trial. Lancet 2020;396(10267):1979-1993. doi:10.1016/s0140-6736 (20)32466-1.

[2] Pfizer. Pfizer and BioNTech Conclude Phase 3 Study of COVID-19 Vaccine Candidate, Meeting All Primary Efficacy Endpoints [updated November 18, 2020]. Available from: https://www.pfizer.com/news/ press-release/press-release-detail/pfizer-and-biontech-concludephase-3-study-covid-19-vaccine. Accessed November 26, 2020.

[3] NIH. Promising Interim Results from Clinical Trial of NIH-Moderna COVID-19 Vaccine [updated November 16, 2020]. Available from: https:// www.nih.gov/news-events/news-releases/promising-interim-resultsclinical-trial-nih-moderna-covid-19-vaccine. Accessed November 26, 2020.

[4] FDA. Pfizer-BioNTech COVID-19 Vaccine [updated December 11, 2020]. Available from: https://www.fda.gov/emergency-preparedness-and-response/coronavirus-disease-2019-covid-19/pfizer-biontech-covid-19-vaccine. Accessed December 13, 2020.

[5] Ledford H, Cyranoski D, Van Noorden R. The UK has approved a COVID vaccine-here's what scientists now want to know. Nature 2020;588(7837):205-206. doi:10.1038/d41586-020-03441-8.

[6] Sugden J, Horner W. U.K. Begins Rollout of Pfizer's Covid-19 Vaccine in a First for the West: Less than a week after the shot was authorized, the first Britons receive doses [updated December 08, 2020]. Available from: https://www.wsj.com/articles/u-k-begins-rollout-of-pfizers-covid-19-vaccine-in-a-first-for-the-west-11607419672. Accessed December 08, 2020.

[7] FDA. Moderna COVID-19 Vaccine [updated December 18, 2020]. Available from: https://www.fda.gov/emergency-preparedness-andresponse/coronavirus-disease-2019-covid-19/moderna-covid-19vaccine. Accessed December 24, 2020.

[8] Marris S. COVID-19: Moderna coronavirus vaccine approved for emergency use in the US [updated December 19, 2020]. Available from: https://news.sky.com/story/moderna-coronavirus-vaccine-approved-for-emergency-use-in-the-us-12166939. Accessed December 24, 2020.

[9] National Academies of Sciences, Engineering, and Medicine. Framework for Equitable Allocation of COVID-19 Vaccine. National Academies Press; 2020.

[10] Emanuel EJ, Persad G, Kern A, Buchanan A, Fabre C, Halliday D, et al. An ethical framework for global vaccine allocation. Science 2020; 369(6509):1309-1312. doi:10.1126/science.abe2803.

[11] Pfizer. Pfizer and BioNTech Announce Vaccine Candidate Against COVID-19 Achieved Success in First Interim Analysis from Phase 3 Study [updated November 09, 2020]. Available from: https://www.pfizer. $\mathrm{com} /$ news/press-release/press-release-detail/pfizer-and-biontechannounce-vaccine-candidate-against. Accessed November 26, 2020.

[12] Knipe DM, Levy O, Fitzgerald KA, Mühlberger E. Ensuring vaccine safety. Science 2020;370(6522):1274-1275. doi:10.1126/science.abf0 357.

[13] The COVID vaccine challenges that lie ahead. Nature 2020;587(7835): 522. doi:10.1038/d41586-020-03334-w.

[14] Anderson RM, Vegvari C, Truscott J, Collyer BS. Challenges in creating herd immunity to SARS-CoV-2 infection by mass vaccination. Lancet 2020;396(10263):1614-1616. doi:10.1016/s0140-6736(20)32318-7.

[15] Ward JK, Alleaume C, Peretti-Watel P, COCONEL Group. The French public's attitudes to a future COVID-19 vaccine: The politicization of a public health issue. Soc Sci Med 2020;265:113414. doi:10.1016/j. socscimed.2020.113414.

[16] Salali GD, Uysal MS. COVID-19 vaccine hesitancy is associated with beliefs on the origin of the novel coronavirus in the UK and Turkey Psychol Med 2020:1-3. doi:10.1017/S0033291720004067.

[17] Lin Y, Hu Z, Zhao Q, Alias H, Danaee M, Wong LP. Understanding COVID-19 vaccine demand and hesitancy: A nationwide online survey in China. PLoS Negl Trop Dis 2020;14(12):e0008961. doi:10.1371/journal.pntd.0008961. 
[18] Shaman J, Galanti M. Will SARS-CoV-2 become endemic? Science 2020;370(6516):527-529. doi:10.1126/science.abe5960.

[19] WHO. Coronavirus disease (COVID-2019) situation reports [updated
December 22, 2020]. Available from: https://www.who.int/emergencies/diseases/novel-coronavirus-2019/situation-reports/. Accessed December 24, 2020. 